\title{
Editorial
}

\section{Millimetre-Wave Antennas and Systems for the Future 5G}

\author{
Masood Ur-Rehman, ${ }^{1}$ Qammer Hussain Abbasi, ${ }^{2,3}$ Atiqur Rahman, ${ }^{4}$ \\ Imdad Khan, ${ }^{5}$ Hassan Tariq Chattha, ${ }^{5}$ and Mohammad Abdul Matin ${ }^{6}$ \\ ${ }^{1}$ Centre for Wireless Research, University of Bedfordshire, Luton LU1 3JU, UK \\ ${ }^{2}$ Texas A \& $M$ University at Qatar, Doha, Qatar \\ ${ }^{3}$ School of Engineering, University of Glasgow, Glasgow G12 8QQ, UK \\ ${ }^{4}$ Department of Electrical \& Computer Engineering, North South University, Dhaka, Bangladesh \\ ${ }^{5}$ Department of Electrical Engineering, Faculty of Engineering, Islamic University in Madinah, Al-Madinah, Saudi Arabia \\ ${ }^{6}$ Department of Electrical and Electronic Engineering, Institute Teknologi Brunei, Gadong, Brunei Darussalam
}

Correspondence should be addressed to Masood Ur-Rehman; masood.urrehman@beds.ac.uk

Received 4 April 2017; Accepted 5 April 2017; Published 10 April 2017

Copyright ( 2017 Masood Ur-Rehman et al. This is an open access article distributed under the Creative Commons Attribution License, which permits unrestricted use, distribution, and reproduction in any medium, provided the original work is properly cited.

\section{Introduction}

$5 \mathrm{G}$ is the next technological marvel enabling ubiquitous portable systems for the realization of Internet of Things (IoT). Millimeter-wave frequency range is a prestandardization favorite for the portable $5 \mathrm{G}$ applications. High performing millimeter-wave devices require efficient lowprofile antennas to ensure reliable and interference-free communications. Requirements for increased power, larger bandwidth, higher gain, and insensitivity to the human user presence further complicate the antenna and propagation aspects. Enhanced techniques for multiplexing, interference mitigation, scheduling, and radio resource allocation work alongside the antenna design to realize efficient millimeterwave systems delivering seamless and optimal performance. Being a newly developed area, simulation techniques also need to be revisited to ensure high level of accuracy of millimeter-wave antennas and systems. It solicits novel ideas and innovative solutions for the antenna design and system development.

This special issue is intended to reflect current research trends and novel approaches to address the issues of antenna design and propagation for $5 \mathrm{G}$ enabled millimeterwave applications. Particular emphasis has been put in the antenna design and measurement methods, MIMO antenna systems, and beamforming techniques; solutions for advanced millimeter-wave operational scenarios including device-to-device communications, multiplexing, spectrum cognition, and interference mitigation have also received particular emphasis.

\section{Contributions}

The special issue consists of 7 contributions covering a variety of antenna design and MIMO techniques.

In "Multiband Split-Ring Resonator Based Planar Inverted-F Antenna for 5G Applications" by M. K. Ishfaq et al., the authors present design and realization of a novel multiband antenna for 5G applications. The antenna is composed of a PIFA, an inverted-L parasitic element, a rectangular shaped parasitic element, and a split-ring resonator etched on the top plate of the PIFA. The antenna covers three frequency bands at $6 \mathrm{GHz}(5-7 \mathrm{GHz}), 10 \mathrm{GHz}$ $(9-10.8 \mathrm{GHz})$, and $15 \mathrm{GHz}(14-15 \mathrm{GHz})$, each with more than $1 \mathrm{GHz}$ impedance bandwidth. It exhibits peak gains of $3.4 \mathrm{dBi}, 4.9 \mathrm{dBi}$, and $5.85 \mathrm{dBi}$, respectively, at the three bands.

In "Millimeter-Wave Microstrip Antenna Array Design and an Adaptive Algorithm for Future 5G Wireless Communication Systems" by C.-N. Hu et al., the authors present a high gain millimeter-wave Low-Temperature Cofired Ceramic microstrip antenna array having a compact, simple, and low-profile structure. Significant level of interference mitigation is achieved by incorporating minimum mean square error adaptive algorithm with the proposed antenna. 
The simulated antenna performance is validated through measurements and demonstrates a return loss of $\geq 15$ and a peak gain of $\geq 6.5 \mathrm{dBi}$ at $37.5-39 \mathrm{GHz}$ frequency band.

In "Enhanced Next Generation Millimeter-Wave Multicarrier System with Generalized Frequency Division Multiplexing" by H. Shimodaira et al., the authors discuss a new Generalized Frequency Division Multiplexing frame to comply it with IEEE 802.11ad standard and extend the intercarrier interference reduction technique to be used for the wireless systems using this standard. The proposed method exhibits good Peak-to-Average Power Ratio and throughput performance simultaneously while reducing the computational costs by $83 \%$. This method is applicable to other mmWave standards in IEEE 802.1lay and 5G mmWave cellular networks as well.

In "Pilot Contamination Mitigation via a Novel TimeShift Pilot Scheme in Large-Scale Multicell Multiuser MIMO Systems" by Z. Luo et al., the authors propose a novel timeshift pilot scheme with asymptotic channel orthogonality to mitigate the pilot contamination in Large-Scale Multicell Multiuser MIMO systems. The proposed method considers the users within a cell to transmit the same pilot sequence in a time-shift manner enabling channel state information to be estimated without contamination. For different cells, pilot sequences are considered to be mutually orthogonal. Analysis of channel coefficient estimation, uplink data detection, and downlink data transmission steps shows that the proposed method alleviates the pilot contamination problem and improves the performance of the system significantly compared with the popular orthogonal pilots.

In "Connectivity Analysis of Millimeter-Wave Device-toDevice Networks with Blockage" by H. Jung and I.-H. Lee, the authors analyse the impact of blockage of the LoS on the direct and indirect device-to-device (D2D) communications in millimeter-wave cellular networks. The connectivity performance in terms of the probability to achieve a fully connected network and average number of reliably connected devices is evaluated. It is observed that both connectivity performance metrics decrease with an increase in network size and the blockage. The authors recommend use of a hybrid of direct and indirect communication to improve the two parameters by $35 \%$ based on simulation results.

In "Joint User Scheduling and MU-MIMO Hybrid Beamforming Algorithm for mmWave FDMA Massive MIMO System" by J. Jiang and D. Kong, the authors propose a joint user scheduling and multiuser hybrid beamforming algorithm for downlink massive MIMO Orthogonal Frequency Division Multiple Access (OFDMA) system. The users with identical optimal beams form an OFDMA user group and multiplex the entire frequency resource. The base station then allocates the frequency resources to each member of the group. Each of the user groups is regarded as a virtual user enabling support of arbitrary MU-MIMO user selection and beamforming algorithms. In the proposed technique, the analog beamforming vectors employ the best beam of each selected MU-MIMO user and the digital beamforming algorithm is solved by weight MMSE to acquire the maximum gain and mitigate interuser inference.
In "A Novel Simulator of Nonstationary Random MIMO Channels in Rayleigh Fading Scenarios" by Q. Zhu et al., the authors propose a new model for simulations of nonstationary MIMO Rayleigh fading channels in time-variant scattering environments. The simulator is based on the sum-of-sinusoids method to achieve low complexity and implementation simplicity. In order to reproduce realistic time varying statistics for dynamic channels, an efficient method to update frequency parameters is also proposed. Comparative analysis with theoretical models indicates that the proposed method generates channel statistics with good level of accuracy.

These papers provide a good overview of current research and development activities going on in the selected areas of millimeter-wave systems. The editors hope that this special issue will benefit the scientific community and contribute to the knowledge base.

\section{Acknowledgments}

The editors would like to take this opportunity to applaud the contribution of the authors to this special issue. Efforts of the reviewers to enhance the quality of the manuscripts are also much appreciated.

Masood Ur-Rehman

Qammer Hussain Abbasi Atiqur Rahman Imdad Khan

Hassan Tariq Chattha

Mohammad Abdul Matin 


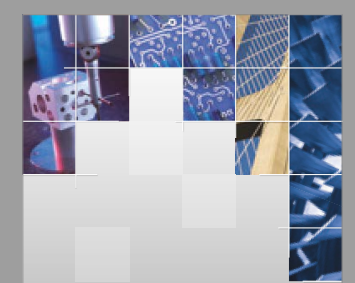

\section{Enfincering}
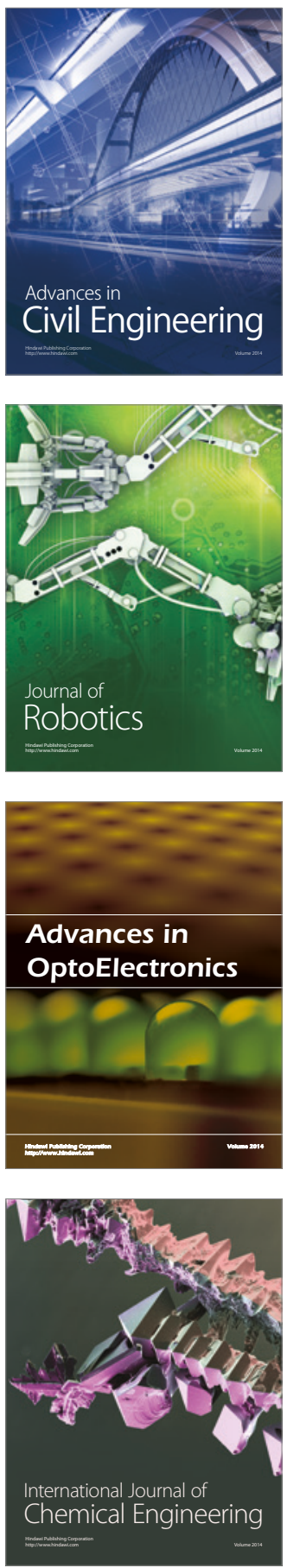

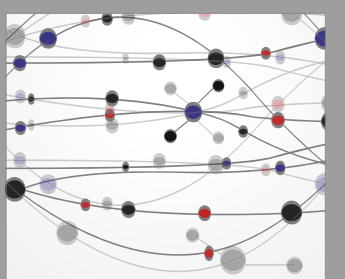

The Scientific World Journal

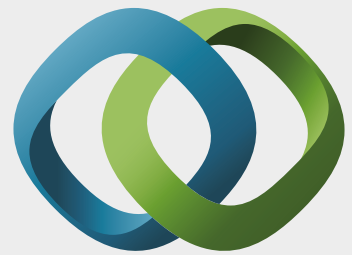

\section{Hindawi}

Submit your manuscripts at

https://www.hindawi.com
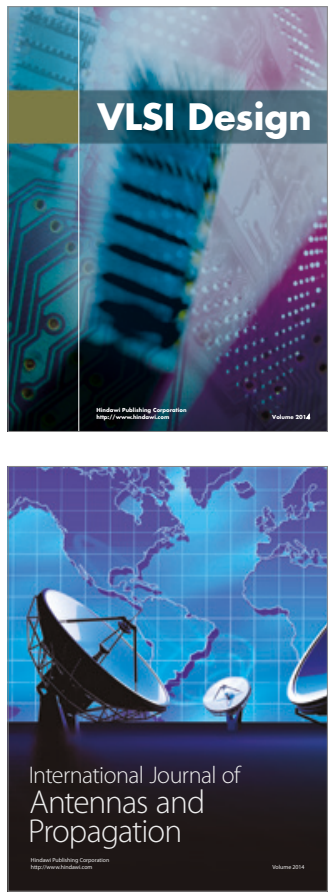

\section{Rotating}

Machinery
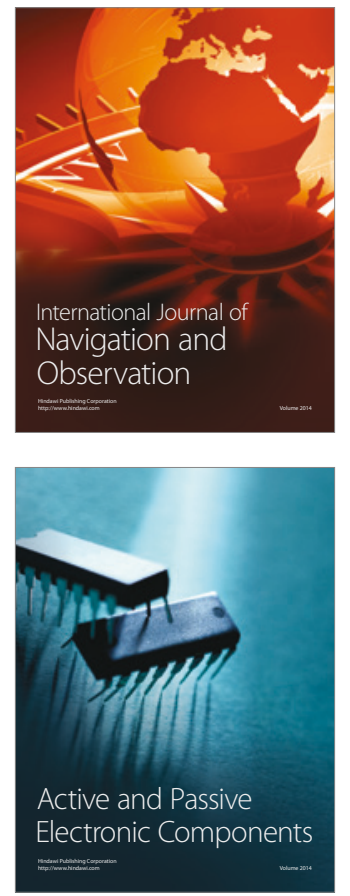
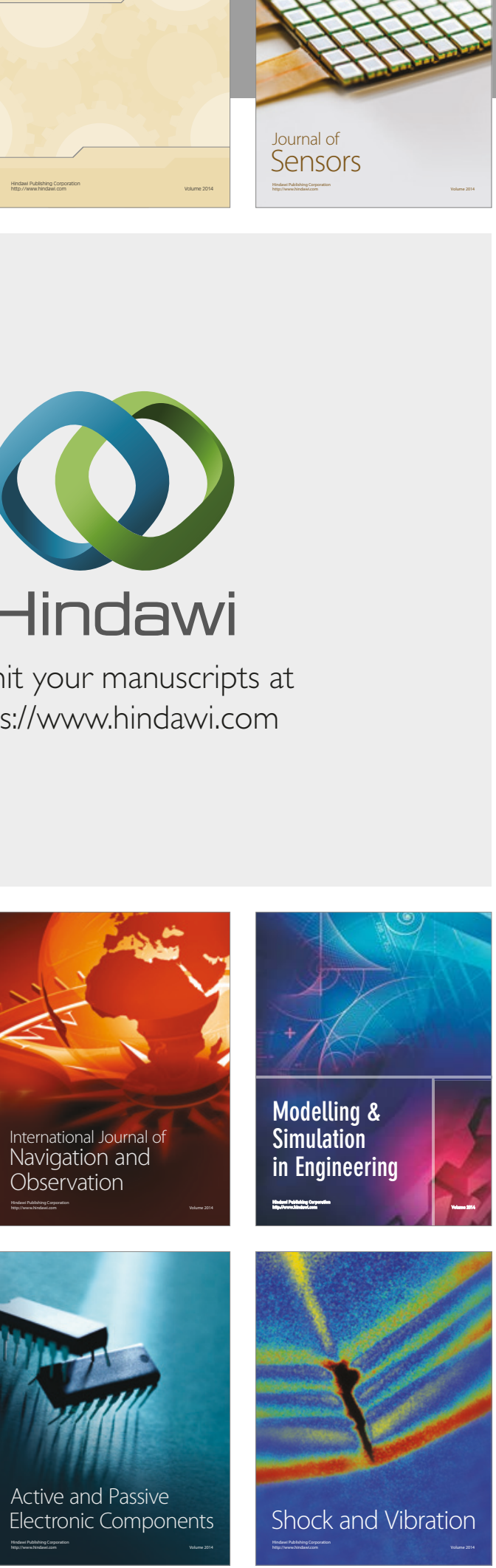
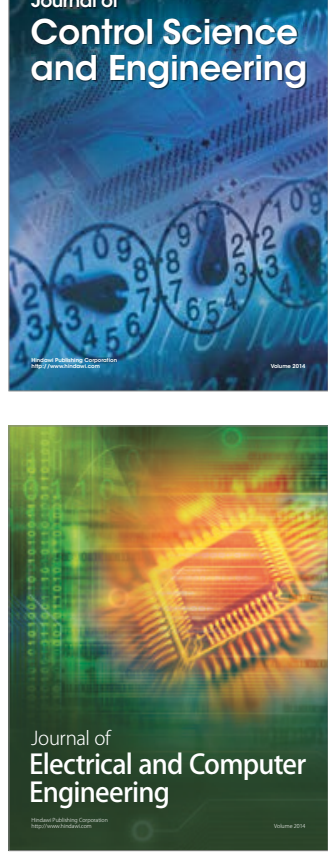

Distributed

Journal of

Control Science

and Engineering
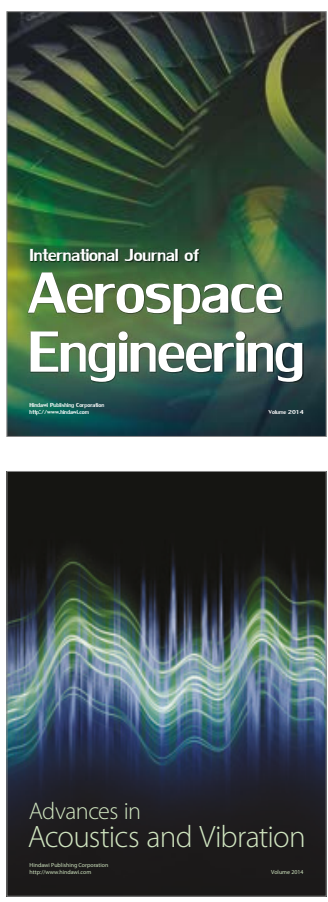

Sensor Networks 